\title{
Expression of Col1a1, Col1a2 and procollagen I in germ cells of immature and adult mouse testis
}

\author{
Zuping He, Lixin Feng, Xiaodong Zhang, Yixun Geng, Daniela A Parodi, Carlos Suarez-Quian \\ and Martin Dym \\ Department of Cell Biology, Georgetown University Medical Center, 3900 Reservoir Road NW, Washington, \\ District of Columbia 20057, USA
}

Correspondence should be addressed to M Dym; Email: dymm@georgetown.edu

\begin{abstract}
The objective of this study was to compare the expression of Col1a1, Col1a2, and procollagen I in the seminiferous tubules of immature and adult mice and to characterize the cellular expression pattern of procollagen I in germ cells during spermatogenesis in order to provide necessary groundwork for further functional studies in the process of spermatogenesis. Microarray analysis demonstrated that Col1a1 and Col1a2 were abundantly expressed in the seminiferous tubules of 6-day-old mice compared with 60-day-old mice, and the expression levels of Col1a1 and Col1a2 mRNA were validated using a semi-quantitative RT-PCR assay. Western blot analysis further confirmed that procollagen I was expressed at a higher level in the seminiferous tubules of 6-day-old mice compared with 60-day-old mice. Immunohistochemical analysis revealed that type A spermatogonia were positive for procollagen I in the testis of 6-day-old mice, whereas Sertoli cells were negative for this protein. The in vivo procollagen I staining in type A spermatogonia was corroborated in spermatogonia exhibiting a high potential for proliferation and the ability to form germ cell colonies in in vitro culture. Moreover, procollagen I was also detected in type A spermatogonia, intermediate spermatogonia, type B spermatogonia, and preleptotene spermatocytes in the adult mouse testes, but positive staining disappeared in more differentiated germ cell lineages detaching from the basement membrane, including leptotene spermatocytes, pachytene spermatocytes, round spermatids and elongated spermatids. These data suggest that Col1a1, Col1a2 and procollagen I are associated with type A spermatogonia and play a potential role in mediating the detachment and migration of germ cells during spermatogenesis.

Reproduction (2005) 130 333-341
\end{abstract}

\section{Introduction}

The seminiferous epithelium of the testis rests on a thin zone of extracellular matrix known as the basement membrane (Dym 1994). Within the seminiferous tubules, type A spermatogonia, intermediate spermatogonia, type B spermatogonia and preleptotene spermatocytes rest on this basement membrane in the basal compartment, and more differentiated spermatocytes and spermatids, including leptotene spermatocytes, pachytene spermatocytes, round spermatids and elongated spermatids reside in the adluminal compartment, no longer having a direct contact with the basement membrane (Dym \& Fawcett 1970, Dym \& Cavicchia 1977). Spermatogenesis is the process of morphological and functional differentiation of type A spermatogonia into mature spermatids. An important aspect of spermatogenesis involves the detachment of germ cells from the basement membrane and their subsequent migration towards the tubule lumen. However, the molecular mechanisms, especially the specific genes that account for the detachment and migration of germ cells during spermatogenesis, remain unclear. Microarray technology has the potential to measure simultaneously the expression levels of tens of thousands of genes in a biological sample (Lockhart et al. 1996, Schena et al. 1996), and thus it could be useful to characterize the change in expression of genes associated with differentiation and development during spermatogenesis.

Collagens belong to a family of extracellular matrix proteins that play an important role in developmental processes and are necessary structural components of tissues and organs, including lung, skin, bone and blood vessels (Arden et al. 1993, Koch et al. 2003, Boland et al. 2004, 
Chanut-Delalande et al. 2004). Type I collagen, a major collagenous component, plays a role in neural crest cell migration (McCarthy \& Hay 1991) and may be related to the regulation of cellular differentiation. There is evidence demonstrating that the terminal differentiation of keratinocytes involves the loss of adhesion to extracellular matrix components including type I collagen (Adams \& Watt 1990), and type I collagen mRNA was also shown to be down-regulated during mesenchymal stem cell differentiation into chondrocyte-like cells (Chen et al. 2004). Moreover, type I collagen was used as a dedifferentiation marker for human nasal chondrocytes and fetal bovine chondrocytes, and a switch of collagen production from type II and type IX collagen to type I collagen occurred during the proliferation of chondrocytes (Ronziere et al. 1997, Malda et al. 2004). Procollagen I, a precursor of type I collagen, is a trimer consisting of two $\alpha 1$ chains and one $\alpha 2$ chain whose sequences are encoded by two different genes, namely Col1a1 and Col1a2 respectively. Gene targeting technology has been used to disrupt Col1a1 in mesenchymal progenitor cells of patients with osteogenesis imperfecta caused by mutations in Col1a1 and Col1a2 (Chamberlain et al. 2004). This technology leads to an improvement of collagen processing, stability and structure. However, the possible roles of Col1a1 and Col1a2 in the process of spermatogenesis and the cellular expression pattern of procollagen $I$ in the seminiferous tubules remain to be elucidated.

We hypothesized that Col1a1, Col1a2 and procollagen I may be involved in the regulation of type A spermatogonia and may play a potential role in mediating the adhesion and migration of germ cells within the seminiferous tubules during spermatogenesis. Thus, the aims of this study were, first, to compare the expression of Col1a1 mRNA, Col1a2 mRNA and procollagen I in the seminiferous tubules of 6-day-old mice and adult mice using microarray analysis, semi-quantitative RT-PCR and Western blot assay, and, secondly, to characterize the cellular expression pattern of procollagen I within the seminiferous tubules of immature and adult mice utilizing immunohistochemistry and immunocytochemistry.

\section{Materials and Methods}

\section{Animals}

Balb-C adult male mice and mothers with male pups were obtained from the Charles River Laboratories, Inc. (Wilmington, MA, USA). The animals were maintained in the Research Resource Facility at Georgetown University Medical Center and adult mice were provided with a standard diet. All animal care procedures were carried out pursuant to the National Research Council's Guide for the Care and Use of Laboratory Animals, and the experimental protocols employed in this study were approved by the Animal Care and Use Committee of Georgetown University.

\section{Microarray analysis}

The seminiferous tubules were isolated from the testes of 6-day-old and 60-day-old mice using mechanical dissociation and enzymatic digestion according to a method previously described by our laboratory (Bellve et al. 1977, Dym et al. 1995). Total RNA was extracted from the seminiferous tubules utilizing the Trizol reagent (Invitrogen, San Diego, CA, USA) pursuant to the manufacturer's protocol, and purified using the RNeasy Mini kit (QIAGEN Inc., Valencia, CA, USA). The concentration and purity of total RNA and biotin-labeled cRNA were evaluated by measuring the $260 / 280 \mathrm{~nm}$ ratios, and the integrity of total RNA and the quality of biotin-labeled cRNA were assessed by $1.0 \%$ denaturing agarose gel electrophoresis. Total RNA with a 260/280 nm ratio of approximately 2.0 was used to generate biotin-labeled cRNA target for the Murine GeneChip (Affymetrix, Santa Clara, CA, USA). Ten micrograms total RNA were reverse-transcribed into single strand cDNA that was subsequently converted to double strand cDNA and purified by the use of the GeneChip Sample Cleanup Module kit (Affymetrix). The purified double strand cDNA served as a template to synthesize biotin-labeled cRNA using the BioArray High Yield RNA Transcription Labeling kit (Enzo Life Sciences, Inc., Farmingdale, NY, USA), and the biotin-labeled cRNA was purified using the GeneChip Sample Cleanup Module kit (Affymetrix) and fragmented to 35-200 base fragments according to the Affymetrix protocol. The Murine Genome U74A Array from Affymetrix was loaded with $200 \mu$ l of the hybridization cocktail containing $15 \mu \mathrm{g}$ of the fragmented biotin-labeled cRNA and then hybridized for $16 \mathrm{~h}$ at $45^{\circ} \mathrm{C}$. After hybridization, the array was washed, stained with streptavidin phycoerythrin using the Affymetrix Genechip Fluidics Workstation 400, and scanned on a Hewlett-Packard Gene Array scanner (Hewlett-Packard Co., Palo Alto, CA, USA). Finally, raw data were generated and analyzed using the GeneSpring 5.0 software (Silicon Genetics, Redwood City, CA, USA). The microarray analysis was repeated three times with 48 6-day-old mice (96 testes) in each experiment and the data are presented as mean \pm standard deviation.

\section{RNA extraction and semi-quantitative RT-PCR assay}

A semi-quantitative RT-PCR assay was performed to confirm the expression of Col1a1 and Col1a2 mRNA in 6-day-old and 60-day-old mice. Total RNA was extracted from the seminiferous tubules using the Trizol reagent (Invitrogen), and $1 \mu \mathrm{g}$ total RNA was reverse-transcribed into the first strand CDNA in a reaction primed by oligo (dT)12-18 primer using Superscript II reverse transcriptase (Invitrogen). Two microliters of the first strand cDNA were used as template for the PCR reactions using Taq polymerase (Life Technologies, Inc.). The PCR reaction started at $94^{\circ} \mathrm{C}$ for $2 \mathrm{~min}$, followed by 35 cycles $\left(94^{\circ} \mathrm{C}\right.$ for $30 \mathrm{~s}$, $56^{\circ} \mathrm{C}$ for $45 \mathrm{~s}$, and $72{ }^{\circ} \mathrm{C}$ for $45 \mathrm{~s}$ ), and ended with a 7 -min incubation at $72^{\circ} \mathrm{C}$. The sense and anti-sense primers of 
Col1a1 and Col1a2 were designed using Primer Premier 5.0 software (PREMIER Biosoft International, Palo Alto, CA, USA). The primers used for PCR amplification were as follows: Col1a1, forward 5'-GACGCCATCAAGGTCTACTG-3' and reverse 5'-ACGGGAATCCATCGGTCA-3', product size $154 \mathrm{bp}$; Col1a2, forward 5'-GGAGGGAACGGTCCACGAT-3' and reverse 5'-GAGTCCGCGTATCCACAA-3', product size $133 \mathrm{bp}$; GAPDH, forward 5'-GCATTGTGGAAGGGCTCA-3' ${ }^{\prime}$ and reverse 5'-GGGTAGGAACACGGAAGG-3', product size $207 \mathrm{bp}$. GAPDH was used as a positive control and the PCR products were separated by electrophoresis in $1.2 \%$ agarose gels.

\section{Western blot analysis}

The seminiferous tubules were isolated from 6-day-old and 60-day-old mice as described above and lysed in RIPA lysis buffer containing Tris-buffered saline (TBS), 1\% Nonidet P-40, 0.5\% sodium deoxycholate, 0.1\% SDS, and $0.004 \%$ sodium azide (Santa Cruz Biotechnology Inc., Santa Cruz, CA, USA). Forty micrograms total protein from each sample were used for SDS-PAGE and SeeBlue Plus2 pre-stained standard marker (Invitrogen, Carlsbad, CA, USA) was used as the molecular mass standard for the proteins. Samples were resolved for $1 \mathrm{~h}$ in a minigel apparatus (Bio-Rad Laboratories, Inc., Hercules, CA, USA) and transferred to nitrocellulose membranes. Ponceau stain (Sigma, St Louis, MO, USA) was used to confirm equal loading and transfer of the proteins. The membranes were washed with TBS containing 0.1\% Tween (TBS-T) and blocked with $4 \%$ nonfat dry milk in $10 \mathrm{mM}$ Tris- $\mathrm{HCl}$ for $1 \mathrm{~h}$ at room temperature. Subsequently, the membranes were washed 3 times with TBS-T and incubated with antitype I collagen (EMD Bioscience Inc, La Jolla, CA, USA) at 1:200 dilution in TBS-T containing 1\% BSA for $1 \mathrm{~h}$. After extensive washes with TBS-T, the membranes were incubated with peroxidase-conjugated anti-rabbit IgG (Santa Cruz Biotechnology Inc.) at a 1:5000 dilution for $45 \mathrm{~min}$. The proteins were detected using the ECL system (Amersham Pharmacia Biotech Inc, Piscataway, NJ, USA) and exposed to autoradiography films for $1 \mathrm{~min}$. The membranes were reused for detection of $\alpha$-actin using an antibody to $\alpha$-actin (Sigma).

\section{Immunohistochemistry and immunocytochemistry}

To prepare sections, testes from 6-day-old and adult mice were fixed in $4 \%$ paraformaldehyde, washed with phosphate buffered saline (PBS), and dehydrated through a series of graded alcohols. The testes were then embedded in paraffin at $60^{\circ} \mathrm{C}$ overnight and sections were cut for immunohistochemical analysis.

For immunofluorescent analysis, testis sections from 6-day-old mice were dewaxed in xylene, rehydrated through a series of graded alcohols, fixed in cold methanol for 10 min, penetrated with $0.10 \%$ Nonidet P-40 for $20 \mathrm{~min}$, and blocked with $10 \%$ normal goat serum. After extensive washes with PBS, the sections were incubated with primary antibody to type I collagen (EMD Bioscience Inc.) for $1 \mathrm{~h}$ at $34^{\circ} \mathrm{C}$. After 3 washes in PBS, the testis sections were incubated with fluorescein (FITC)-conjugated IgG for $30 \mathrm{~min}$ at $34^{\circ} \mathrm{C}$ and washed 3 times with PBS. $4^{\prime}, 6^{\prime}$-Diamidino-2-phenylindole (DAPI) was used to stain the nuclei of the cells in the testis, and the sections were observed for epifluorescence using an Olympus Fluoview 500 Laser Scanning Microscope (Olympus, Melville, NY, USA).

Immunofluorescent analysis of the cells derived from the seminiferous tubules of 6-day-old mice was performed according to the methods mentioned above, except that neither paraffin removal nor rehydration was required. Primary antibodies used here included germ cell nuclear antigen (GCNA), a gift from Dr George C Enders, GATA-4 (Santa Cruz Biotechnology Inc.), type I collagen (EMD Bioscience Inc.), and glial cell line-derived neurotropic factor family receptor alpha-1 (GFR $\alpha-1)$ (Santa Cruz Biotechnology Inc.). The second antibodies used were rhodamine-labeled IgG or FITC-conjugated IgG.

For the immunocytochemical assay of spermatogonia in vitro, the seminiferous tubules were isolated from 6-dayold mice and grown on glass coverslips in 6-well plates. The cells derived from the seminiferous tubules attached to the glass coverslips and spread approximately $24 \mathrm{~h}$ after plating; they were fixed with cold acetone, blocked with $10 \%$ normal goat serum, and incubated with anti-type I collagen (EMD Bioscience Inc.) for $1 \mathrm{~h}$ at $34^{\circ} \mathrm{C}$. After extensive washes in PBS, the cells were incubated with the biotinylated second antibody for $20 \mathrm{~min}$. Next, streptavidin-peroxidase enzyme conjugate was added to the cells and reaction product was generated by addition of diaminobenzidine (DAB) as the chromogen. Replacement of the primary antibody with PBS served as a negative control.

Immunohistochemical analysis of the testis sections of adult mice was carried out using the DAB kit (HistostainPlus; Zymed Laboratories, Inc., San Francisco, CA, USA) according to the manufacturer's instructions. Anti-type I collagen (EMD Bioscience Inc.) was used at a dilution of 1:50 for incubation with testis sections for $1 \mathrm{~h}$ at $34^{\circ} \mathrm{C}$. After immunostaining, tissue sections were counterstained with hematoxylin and examined under a light microscope. Replacement of the primary antibody with PBS was used as a negative control.

\section{Results}

\section{Microarray analysis of Col1a1 and Col1a2 mRNA expression in the seminiferous tubules derived from 6-day-old mice and 60-day-old mice}

Using microarray analysis, we discovered that 7757 gene transcripts, including Col1a1 and Col1a2, were present in the seminiferous tubules of both 6-day-old and 60-day-old mice. Col1a1 and Col1a2 were abundantly expressed in the seminiferous tubules of 6-day-old mice, but their 
Table 1 Microarray analysis of Col1a2 and Col1a2 mRNA expression in the seminiferous tubules of 6-day-old and 60-day-old mice.

\begin{tabular}{lcc}
\hline & \multicolumn{2}{c}{ Signal intensity } \\
\cline { 2 - 3 } Gene & 6-day-old mice & 60-day-old mice \\
\hline Col1a1 & $12152 \pm 130$ & $297 \pm 12$ \\
Col1a2 & $6234 \pm 72$ & $283 \pm 10$ \\
\hline
\end{tabular}

mRNA expression decreased to an apparently low level in the seminiferous tubules of 60-day-old mice (Table 1).

\section{Expression of Col1a1, Col1a2, and procollagen I in the seminiferous tubules of 6-day-old mice and 60-day-old mice detected by semi-quantitative RT-PCR and Western blot analysis}

To validate the microarray data, we used a semi-quantitative RT-PCR analysis to investigate the expression of Col1a1 and Col1a2 mRNA in the seminiferous tubules derived from 6-day-old and 60-day-old mice. Col1a1 (154-bp amplicon) and Col1a2 (133-bp amplicon) mRNA were detected in the seminiferous tubules of both immature and adult mice. Furthermore, the mRNA for both genes was expressed at apparently higher levels in the tubules of 6-day-old mice compared with 60-day-old mouse tubules (Fig. 1A), results that were consistent with their expression changes in the seminiferous tubules of immature and mature mice observed with the microarray analysis.

We next determined the protein expression of procollagen I in the seminiferous tubules of immature and adult mice using Western blot analysis. The antibody to type I collagen was made against the triple helical portion and thus it is able to recognize procollagen I. We found that procollagen I was present in the seminiferous tubules of both 6-day-old mice and 60-day-old mice. Moreover, procollagen I was expressed at a higher level in the seminiferous tubules of immature mice compared with adult mice (Fig. 1B).

\section{Characterization of the cellular expression pattern of procollagen I in testis sections and in cultured germ cell colonies of immature mice}

We further examined the cellular expression pattern of procollagen I in testis sections of 6-day-old mice using immunofluorescent staining. In the seminiferous epithelium of 6-day-old mice, only type A spermatogonia and Sertoli cells are present (Dettin et al. 2003). Because the antibody to type I collagen was made against the triple helical portion, it is able to stain procollagen I as well as type I collagen. As shown in Fig. 2A, type A spermatogonia were immunostained positively for procollagen I within the seminiferous tubules of 6-day-old mice whereas no specific immunostaining for procollagen I was observed in Sertoli cells. The cells in the interstitial space also appeared to express procollagen I.

Type A spermatogonia possess a high potential for proliferation and have the ability to form colonies in vitro. Thus, we examined the phenotypic characteristics of the colony cells in vitro, first in the isolated seminiferous tubules when they attached to glass coverslips to permit performing the immunofluorescent procedure, and secondly, approximately $24 \mathrm{~h}$ later when the spermatogonia dissociated from the tubules and formed colonies of cells on a bed of immature Sertoli cells. GCNA, a marker for germ cell lineages (Enders \& May 1994), stained the nuclei of all germ cells present in the tubules attached to the coverslips (Fig. 2B), confirming that these cells were indeed spermatogonia. In contrast, the other cell type found in 6-day-old mouse tubules, the immature Sertoli

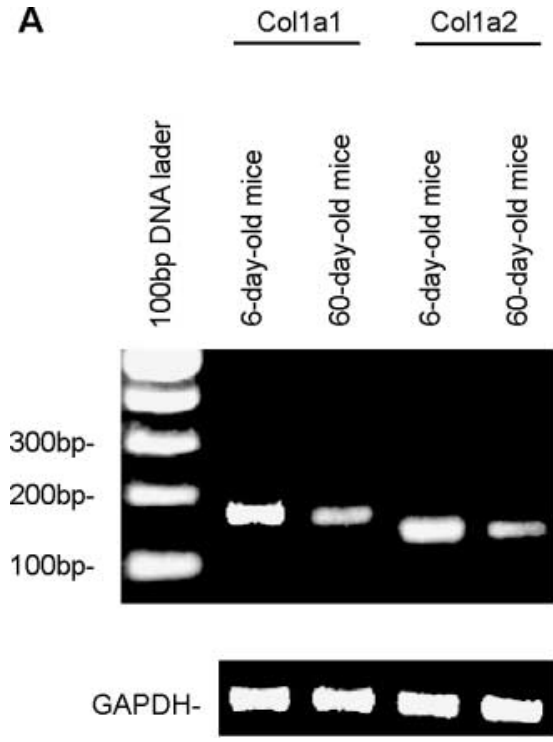

Reproduction (2005) 130 333-341

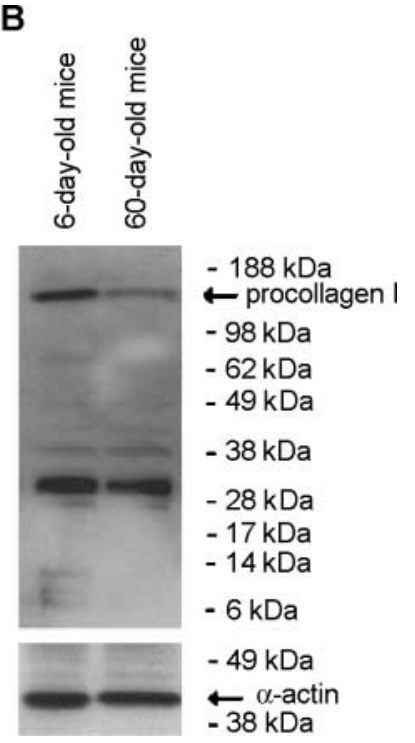

Figure 1 Col1a1 mRNA, Col1a2 mRNA and procollagen I expression in the seminiferous tubules of 6-dayold mice and 60-day-old mice. (A) Semi-quantitative RT-PCR assay showing that Col1a1 and Col1a2 mRNA were expressed at higher levels in the seminiferous tubules of 6-day-old mice compared with 60-day-old mice. GAPDH served as a loading control. (B) Western blot analysis showing that procollagen I was expressed at a higher level in the tubules of 6-day-old mice compared with 60-day-old mice. The Western blot was stripped and re-probed with the antibody against $\alpha$-actin (42 kDa) to check for equal loading of total protein. The data presented in A and $B$ are representative of semi-quantitative RT-PCR and Western blot analysis from three separate isolations of seminiferous tubules obtained from eight different 6-day-old mice and four different 60-day-old mice. 
A

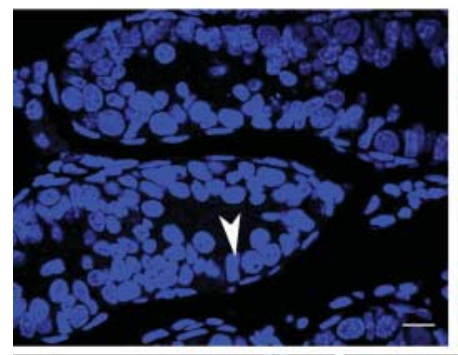

Procollagen I

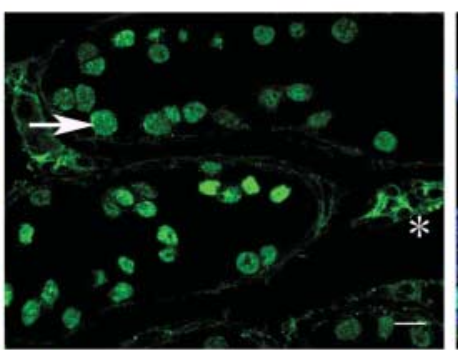

Merged

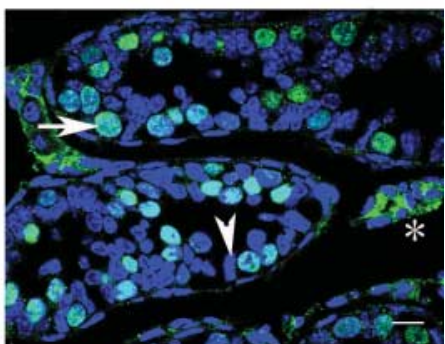

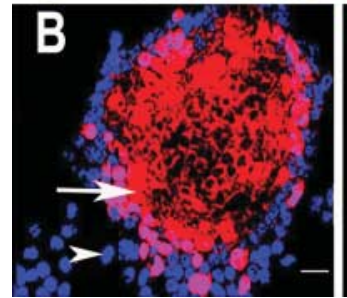
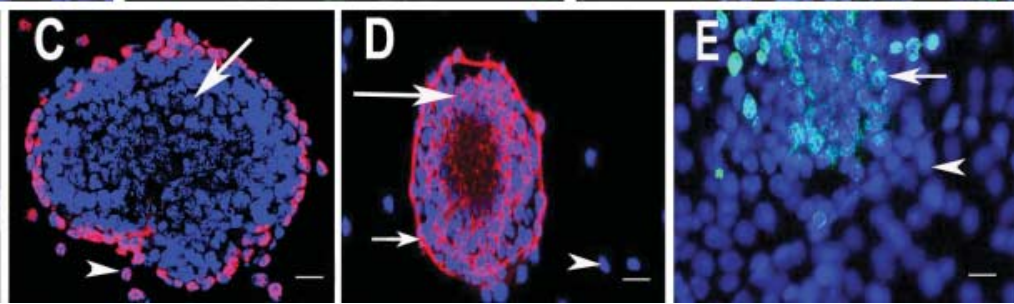

Figure 2 Immunofluorescent analysis of procollagen I in testis sections and cultured germ cell colonies derived from 6-day-old mice. (A) Testis sections ( $5 \mu \mathrm{m}$ thick) were immunostained as described in the Materials and Methods section. Type A spermatogonia (arrows) were positive for procollagen I (green fluorescence), while Sertoli cells (arrowheads) were negative. Interstitial cells on the outside of the seminiferous tubules also showed staining for procollagen I (asterisks). Staining with DAPI (blue fluorescence) was used to identify the nuclei of all cells found in the section. (B to E) Seminiferous tubules were isolated from 6-day-old mice and allowed to attach onto glass coverslips for 6-8 $\mathrm{h}$ before fixation/permeabilization/immunostaining as described in the Materials and Methods section. (B) The nuclei of all spermatogonia (arrow) were stained for GCNA (red fluorescence), whereas Sertoli cells (arrowhead) were negative (indicated by the DAPI blue fluorescence). (C) Sertoli cell nuclei (arrowhead) were positive for GATA-4 (presented as red fluorescence), whereas germ cells (arrow) showed no staining for GATA-4 (exhibiting DAPI blue fluorescence). (D) Type A spermatogonia (large arrow) exhibited positive immunostaining for procollagen I (red fluorescence),

whereas Sertoli cells (arrowhead) were negative and were identified by the DAPI blue fluorescence. A zone of type I collagen (red fluorescence) is noted around the germ cell colony (small arrow). (E) Type A spermatogonia in the germ cell colony also showed positive staining for GFR $\alpha-1$ as indicated by the arrow (green fluorescence), whereas Sertoli cells (arrowhead) were negative. The data presented in A represent immunofluorescent analysis performed in testis sections derived from six different 6-day-old mice. The data presented in B, C, D, and E represent immunofluorescent analysis performed in three separate isolations of seminiferous tubules obtained from six different 6-day-old mice. The scale bar in $A=10 \mu \mathrm{m}$ and the scale bars in $B, C, D$ and $E=20 \mu \mathrm{m}$.

cells, did not stain positively for GCNA but were positive for GATA-4 (Fig. 2C), a specific marker for these cells. Significantly, the spermatogonial colony cells positive for GFR $\alpha-1$ (Fig. 2E), a putative marker for undifferentiated spermatogonia (Dettin et al. 2003), were also robustly positive for procollagen I (Fig. 2D). As is the case in vivo, Sertoli cells did not immunostain for procollagen I.

Twenty-four hours after plating on glass coverslips, seminiferous tubules dissociated into a bed of immature Sertoli cells and a cluster of germ cells that sat as a colony on top of the Sertoli cells. Immunocytochemical assay further revealed that only the spermatogonial cluster cells were immunostained positively for procollagen I (Fig. 3A, B, C). As in the freshly isolated tubules, Sertoli cells were negative for this protein.

\section{Characterization of the cellular expression pattern of procollagen $I$ in the testes derived from adult mice}

In adult mice, the successive steps of germ cell differentiation occur within the context of different cell associations or stages of the cycle of the seminiferous epithelium. We then ascertained the cellular expression pattern of procollagen I in the seminiferous tubules of adult mice at various stages. Procollagen I was expressed in type A spermatogonia (Fig. 4A,B), intermediate spermatogonia (Fig. 4A,B), type B spermatogonia (Fig. 4B), and preleptotene spermatocytes (Fig. 4C). Conversely, procollagen I staining was absent in leptotene spermatocytes, pachytene spermatocytes, round spermatids or elongate spermatids (Fig. 4A, B,C) within the seminiferous tubules derived from adult mice.

\section{Discussion}

During spermatogenesis, germ cells gradually detach from the basement membrane, migrate towards the lumen, and differentiate into spermatocytes that further differentiate into round and elongate spermatids. Other regenerative epithelial systems also contain cells that undergo a similar detachment from their respective basement membranes to differentiate. It has been demonstrated that the proliferation of keratinocytes occurs in the basal layer, which is in contact with basement membrane, and the differentiation of keratinocytes is initiated when they migrate away from the basement membrane (Adams \& Watt 1990). In addition, granule cell precursors stop proliferation and differentiate prematurely once they lose contact 

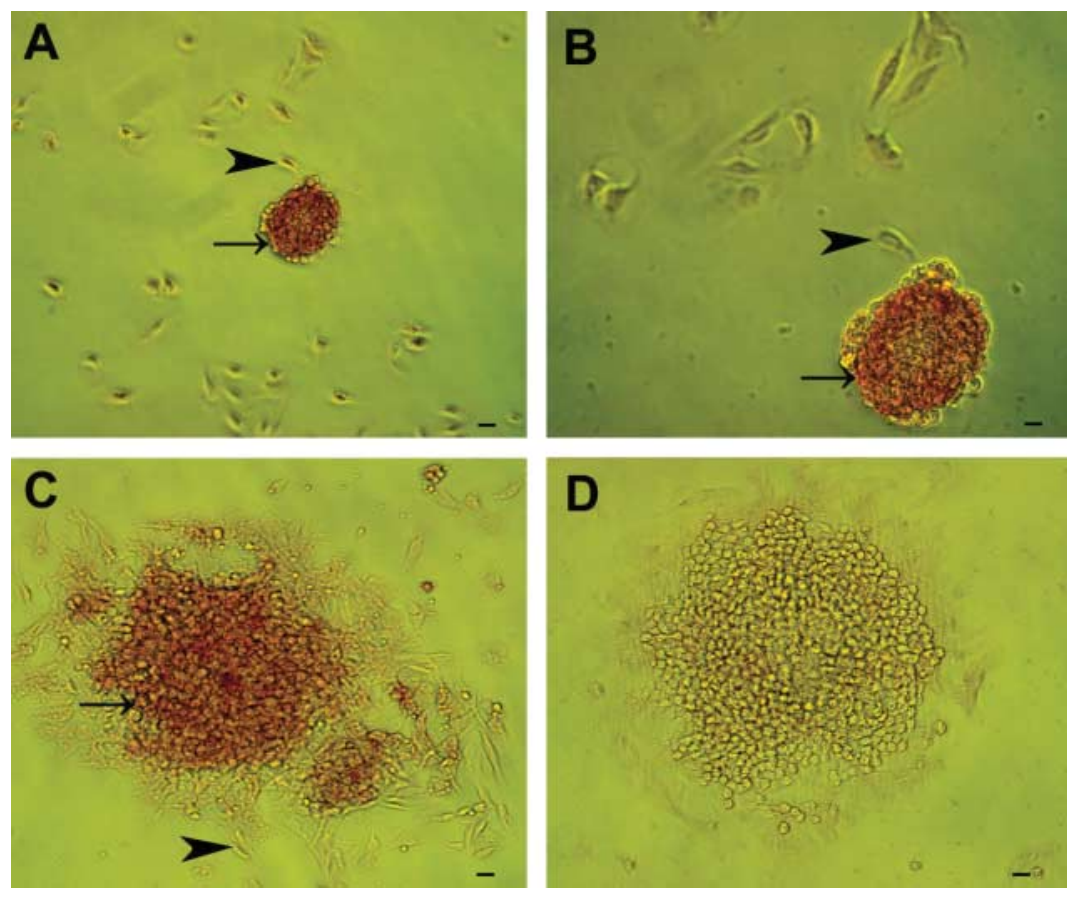

Figure $3 \mathrm{Immunocytochemical} \mathrm{staining} \mathrm{of} \mathrm{procol-}$ lagen I in germ cell clusters formed from the cultured seminiferous tubules of 6-day-old mice.

Seminiferous tubules were allowed to attach onto glass coverslips for approximately $24 \mathrm{~h}$. By this time Sertoli cells migrated away from the tubules and flattened out onto the coverslips. The remaining spermatogonia balled up into cell clusters on the feeder Sertoli cell layer. (A-C) Type A spermatogonia forming the cell clusters were positive for procollagen I (arrow), whereas Sertoli cells were negative (arrowhead). Fig. 3B shows a higher magnification of Fig. 3A. (D) Negative control without primary antibody. The data presented in A, B C, and $D$ represent immunocytochemical analysis performed in three separate isolations of seminiferous tubules obtained from six different 6-day-old mice. Scale bar in $A=30 \mu \mathrm{m}$; scale bar in $B=15 \mu \mathrm{m}$, and the scale bars in $C$ and $\mathrm{D}=25 \mu \mathrm{m}$.
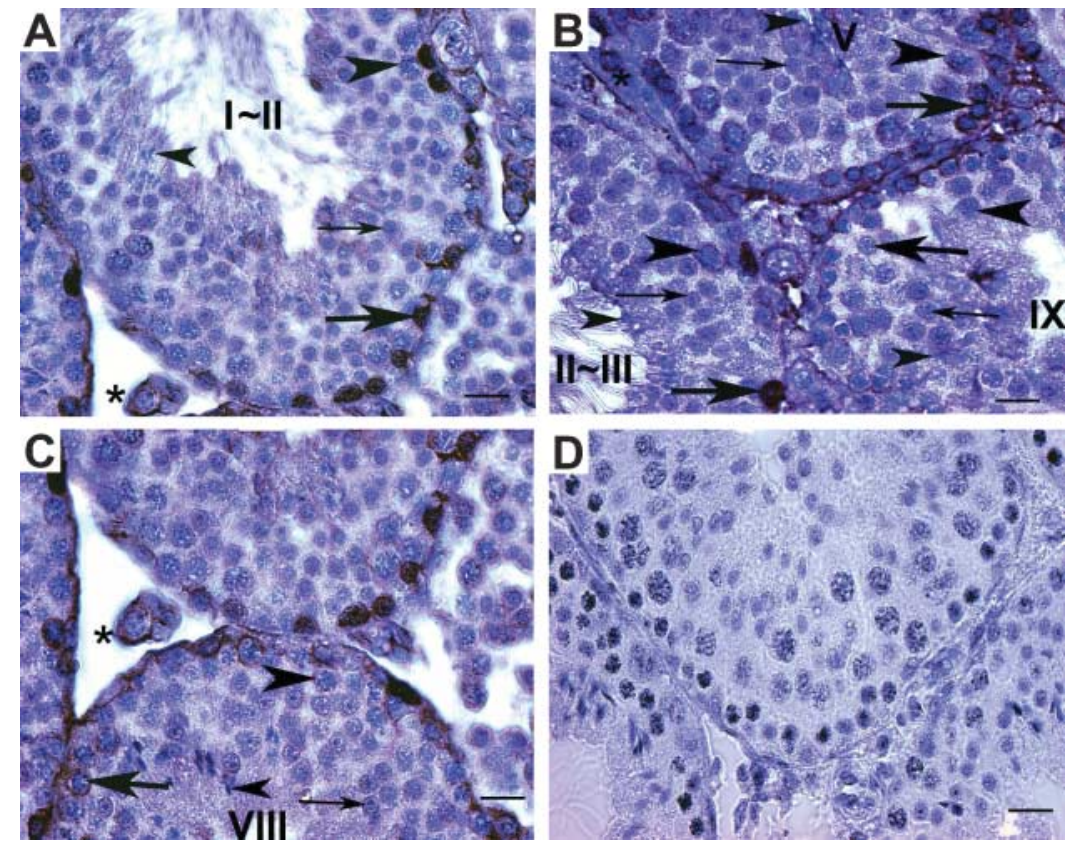

Figure 4 Immunohistochemical assay of procollagen I in testis sections obtained from 60-day-old mice. Testis sections ( $5 \mu \mathrm{m}$ thick) were stained using the DAB kit. (A) Spermatogonia (type A; intermediate) at stage I-II were immunopositive for procollagen I (large arrow). Pachytene spermatocytes (large arrowhead), round spermatids (small arrow) and elongated spermatids (small arrowhead) were negative. Interstitial cells outside the tubules also showed positive staining for procollagen I (asterisk). (B) Spermatogonia at stage II III (type A; intermediate) and type B spermatogonia at stage $\mathrm{V}$ stained positively for procollagen I as indicated by large arrows. Leptotene spermatocytes (large arrow) at stage IX, pachytene spermatocytes (large arrowhead), round spermatids (small arrow) and elongated spermatids (small arrowhead) at stage II III, V and IX were negative for procollagen I. (C) Preleptotene spermatocytes (large arrow) at stage VIII were immunopositive for procollagen I. Pachytene spermatocytes (large arrowhead), round spermatids (small arrow) and elongated spermatids (small arrowhead) at stage VIII were negative for procollagen I. (D) Negative control without primary antibody. The data presented in A, B, C, and D are representative of immunohistochemistry performed in testis sections derived from six different adult mice. Scale bars $=20 \mu \mathrm{m}$. 
with meningeal basement membrane (Blaess et al. 2004). These observations led us to postulate that germ cell detachment from the basement membrane and their migration towards the adluminal compartment must involve universal genes, as well as genes unique to the differentiation process of spermatogenesis.

Here, we have reported for the first time that Col1a1 and Col1a2 are expressed in the seminiferous tubules of both immature and adult mice. We demonstrated, using microarray analysis, that Col1a1 and Col1a2 were abundantly expressed in the seminiferous tubules of immature mice; however, their mRNA expression was apparently at lower levels in the seminiferous tubules of adult mice. The microarray data were corroborated by a semi-quantitative RT-PCR assay and are also in agreement with our group's recent findings that Col1a2 was among the most abundant type A spermatogonial-specific transcripts as seen by the use of serial analysis of gene expression (SAGE) (Wu et al. 2004). In the testis of 6-day-old mice, seminiferous tubules consist of only one type of germ cell, namely primitive type A spermatogonia, and the differentiation of spermatogonia is barely initiated (Dettin et al. 2003). We now confirm that many type A spermatogonia in 6-dayold mice are in an undifferentiated state as indicated by immunofluorescent staining using an antibody to GFR $\alpha-1$. Interestingly, the undifferentiated type A spermatogonia were also strongly positive for procollagen I, suggesting that procollagen I is associated with undifferentiated type A spermatogonia. Moreover, the abundant expression of Col1a1 and Col1a2 mRNA and procollagen I in the seminiferous tubules of 6-day-old mice, together with the substantial decrease in Col1a1 mRNA, Col1a2 mRNA and procollagen I expression in seminiferous tubules of adult mice, likely reflects a relatively lower ratio of undifferentiated type A spermatogonia in the adult mouse testis.

It is worth noting that procollagen I was expressed in type A spermatogonia, intermediate spermatogonia, type B spermatogonia and preleptotene spermatocytes of adult mice. Spermatogonia (type A, intermediate, and type B) have a broad cytoplasmic surface that is flattened onto the basement membrane within the seminiferous tubules, whereas preleptotene spermatocytes have less contact with the basement membrane (Chiarini-Garcia \& Russell 2002). In contrast, leptotene spermatocytes, pachytene spermatocytes, round spermatids and elongated spermatids are no longer in contact with the basement membrane (Dym \& Cavicchia 1978). We discovered that procollagen I staining disappeared in leptotene spermatocytes, pachytene spermatocytes, round spermatids and elongated spermatids. The expression profile of procollagen I in germ cells correlates with their attachment and detachment from the basement membrane. This observation leads us to speculate that procollagen I may be involved in regulating the attachment of these germ cells to the basement membrane within the seminiferous tubules during spermatogenesis, and that loss of procollagen I expression may herald an initiation of subsequent differentiation into later germ cell types. In addition, we demonstrated that Sertoli cells were negative for procollagen I, which was consistent with the observations that Sertoli cells did not release detectable amounts of type I collagen during six days of in vitro culture (Skinner et al. 1985).

It has been demonstrated that transforming growth factor (TGF)- $\beta 2$ enhances the mRNA expression of Col1a1 and Col1a2 in human retinal pigment epithelium (Kimoto et al. 2004). This enhancement of type I collagen production induced by TGF- $\beta 2$ is due to the activation of the p38 mitogen-activated protein kinase signaling pathway (Priglinger et al. 2004). TGF- $\beta 2$ has been shown to promote the attachment and adhesion of retinal pigment epithelial cells to type I collagen (Ghosh et al. 2001), and conversely, interferon-gamma can abrogate the TGF- $\beta 2$ induced Col1a2 transcription and type I collagen synthesis through the activation of the Janus kinase-signal transducer and activator of transcription (JAK-STAT) pathway (Kiger et al. 2001), which is essential for the maintenance of the germ-line stem cell population in Drosophila testis (Jokinen et al. 2004). Interestingly, we found procollagen I was expressed at a higher level in the seminiferous tubules of immature mice compared with adult mice, which was consistent with the expression profiles of Col1a1 and Col1a2 mRNA in the seminiferous tubules of immature and adult mice. This differential gene expression is likely a result of the dilution factor in adult mice which have many more spermatocytes and spermatids that do not express Col1a1 and Col1a2.

Integrins are membrane receptors that connect cells to the extracellular matrix, including collagen, and play a role in regulating cellular migration, proliferation, differentiation and survival (Jokinen et al. 2004). $\beta_{1}$ integrin can pair with different $\alpha$-subunits to form two principal receptors for type I collagen, namely $\alpha_{2} \beta_{1}$ and $\alpha_{1} \beta_{1}$ integrin, and $\alpha_{2} \beta_{1}$ integrin is a functional cellular receptor for type I collagen (Jokinen et al. 2004). Results of numerous studies have demonstrated that $\alpha_{2} \beta_{1}$ integrin is involved in the adhesion of mouse fibroblasts to type I collagen (Cominetti et al. 2004) and $\alpha_{2} \beta_{1}$ integrin rather than $\alpha_{1} \beta_{1}$ integrin mediates cell adhesion to unhydroxylated type I collagen (Perret et al. 2003), indicating that the interaction between cells and unhydroxylated type I collagen is $\alpha_{2} \beta_{1}$-specific. In mesenchymal stem cells, it has been suggested that high levels of type I collagen are required to maintain the cells in an undifferentiated state (Chen et al. 2004). The high levels of Col1a1, Col1a2 and procollagen I that we found in spermatogonia of the immature testis may play a similar role in maintaining the undifferentiated state of the cells. Perhaps as the germ cells lose these genes they proceed towards the differentiated state. Nevertheless, the mechanisms that regulate the interaction of $\alpha_{2} \beta_{1}$ integrin with extracellular matrix that eventually results in the adhesion of spermatogonia and preleptotene spermatocytes to the basement membrane need to be explored further.

In summary, we have demonstrated that Col1a1, Col1a2 and procollagen I are enriched in seminiferous 
tubules of immature mice compared with adult mice. The results suggest that these components may play a role in maintaining type $A$ spermatogonia in an undifferentiated state. We also present here for the first time the distribution of procollagen I within the seminiferous tubules of immature and adult mice and its correlation with the attachment and detachment of germ cells from the basement membrane. The unique distribution pattern of procollagen I in adult mouse testis implies possible roles of Col1a1, Col1a2 and procollagen $I$ in regulating the adhesion of spermatogonia and preleptotene spermatocytes to the basement membrane and the detachment and migration of later spermatocytes and spermatids towards the lumen during spermatogenesis.

\section{Acknowledgements}

We thank Dr George C Enders, Department of Anatomy and Cell Biology, University of Kansas Medical Center, for providing the antibody to GCNA. We are also grateful to Alex Potocki for help with the confocal microscope images. This study was supported by the National Institutes of Health Grant HD 33728 to M Dym. The authors declare that there is no conflict of interest that would prejudice the impartiality of this Scientific work.

\section{References}

Adams JC \& Watt FM 1990 Changes in keratinocyte adhesion during terminal differentiation: reduction in fibronectin binding precedes alpha 5 beta 1 integrin loss from the cell surface. Cell 63 425-435.

Arden MG, Spearman MA \& Adamson IY 1993 Degradation of type IV collagen during the development of fetal rat lung. American Journal of Respiration and Cell Molecular Biology 9 99-105.

Bellve AR, Cavicchia JC, Millette CF, O'Brien DA, Bhatnagar YM \& Dym M 1977 Spermatogenic cells of the prepuberal mouse: isolation and morphological characterization. Journal of Cell Biology 74 68-85.

Blaess S, Graus-Porta D, Belvindrah R, Radakovits R, Pons S, Littlewood-Evans A, Senften M, Guo H, Li Y, Miner JH, Reichardt LF \& Muller U 2004 Beta1-integrins are critical for cerebellar granule cell precursor proliferation. Journal of Neuroscience 24 3402-3412.

Boland ED, Matthews JA, Pawlowski KJ, Simpson DG, Wnek GE \& Bowlin GL 2004 Electrospinning collagen and elastin: preliminary vascular tissue engineering. Frontiers in Biosciences 9 1422-1432.

Chamberlain JR, Schwarze U, Wang PR, Hirata RK, Hankenson KD, Pace JM, Underwood RA, Song KM, Sussman M, Byers PH \& Russell DW 2004 Gene targeting in stem cells from individuals with osteogenesis imperfecta. Science 303 1198-1201.

Chanut-Delalande H, Bonod-Bidaud C, Cogne S, Malbouyres $M$, Ramirez F, Fichard A \& Ruggiero F 2004 Development of a functional skin matrix requires deposition of collagen $\mathrm{V}$ heterotrimers. Molecular and Cell Biology 24 6049-6057.

Chen G, Liu D, Tadokoro M, Hirochika R, Ohgushi H, Tanaka J \& Tateishi T 2004 Chondrogenic differentiation of human mesenchymal stem cells cultured in a cobweb-like biodegradable scaffold. Biochemistry and Biophysics Research Communications 322 50-55.

Chiarini-Garcia H \& Russell LD 2002 Characterization of mouse spermatogonia by transmission electron microscopy. Reproduction $123567-577$.
Cominetti MR, Terruggi $\mathrm{CH}$, Ramos $\mathrm{OH}$, Fox JW, Mariano-Oliveira A, De Freitas MS, Figueiredo CC, Morandi V \& Selistre-de-Araujo HS 2004 Alternagin-C, a disintegrin-like protein, induces vascular endothelial cell growth factor (VEGF) expression and endothelial cell proliferation in vitro. Journal of Biological Chemistry 279 18247-18255.

Dettin L, Ravindranath N, Hofmann MC \& Dym M 2003 Morphological characterization of the spermatogonial subtypes in the neonatal mouse testis. Biology of Reproduction 69 1565-1571.

Dym M 1994 Basement membrane regulation of Sertoli cells. Endocrine Reviews 15 102-115.

Dym M \& Cavicchia JC 1977 Further observations on the blood-testis barrier in monkeys. Biology of Reproduction 17 390-403.

Dym M \& Cavicchia JC 1978 Functional morphology of the testis. Biology of Reproduction 18 1-15.

Dym M \& Fawcett DW 1970 The blood-testis barrier in the rat and the physiological compartmentation of the seminiferous epithelium. Biology of Reproduction 3 308-326.

Dym M, Jia MC, Dirami G, Price JM, Rabin SJ, Mocchetti I \& Ravindranath N 1995 Expression of c-kit receptor and its autophosphorylation in immature rat type A spermatogonia. Biology of Reproduction 52 8-19.

Enders GC \& May JJ 2nd 1994 Developmentally regulated expression of a mouse germ cell nuclear antigen examined from embryonic day 11 to adult in male and female mice. Developmental Biology $163331-340$.

Ghosh AK, Yuan W, Mori Y, Chen S \& Varga J 2001 Antagonistic regulation of type I collagen gene expression by interferon-gamma and transforming growth factor-beta. Integration at the level of p300/CBP transcriptional coactivators. Journal of Biological Chemistry $27611041-11048$.

Jokinen J, Dadu E, Nykvist P, Kapyla J, White DJ, Ivaska J, Vehvilainen P, Reunanen H, Larjava H, Hakkinen L \& Heino J 2004 Integrin-mediated cell adhesion to type I collagen fibrils. Journal of Biological Chemistry 279 31956-31963.

Kiger AA, Jones DL, Schulz C, Rogers MB \& Fuller MT 2001 Stem cell self-renewal specified by JAK-STAT activation in response to a support cell cue. Science $2942542-2545$.

Kimoto K, Nakatsuka K, Matsuo N \& Yoshioka H 2004 p38 MAPK mediates the expression of type I collagen induced by TGF-beta 2 in human retinal pigment epithelial cells ARPE-19. Investigative Ophthalmology and Visual Science 45 2431-2437.

Koch M, Laub F, Zhou P, Hahn RA, Tanaka S, Burgeson RE, Gerecke DR, Ramirez F \& Gordon MK 2003 Collagen XXIV, a vertebrate fibrillar collagen with structural features of invertebrate collagens: selective expression in developing cornea and bone. Journal of Biological Chemistry 278 43236-43244.

Lockhart DJ, Dong H, Byrne MC, Follettie MT, Gallo MV, Chee MS, Mittmann M, Wang C, Kobayashi M, Horton H \& Brown EL 1996 Expression monitoring by hybridization to high-density oligonucleotide arrays. Nature Biotechnology 14 1675-1680.

McCarthy RA \& Hay ED 1991 Collagen I, laminin, and tenascin: ultrastructure and correlation with avian neural crest formation. International Journal of Developmental Biology 35 437-452.

Malda J, van Blitterswijk CA, van Geffen M, Martens DE, Tramper J \& Riesle J 2004 Low oxygen tension stimulates the redifferentiation of dedifferentiated adult human nasal chondrocytes. Osteoarthritis Cartilage 12 306-313.

Perret S, Eble JA, Siljander PR, Merle C, Farndale RW, Theisen M \& Ruggiero F 2003 Prolyl hydroxylation of collagen type I is required for efficient binding to integrin alpha 1 beta 1 and platelet glycoprotein VI but not to alpha 2 beta 1. Journal of Biological Chemistry $27829873-29879$.

Priglinger SG, Alge CS, Neubauer AS, Kristin N, Hirneiss C, Eibl K, Kampik A \& Welge-Lussen U 2004 TGF-beta2-induced cell surface tissue transglutaminase increases adhesion and migration of RPE cells on fibronectin through the gelatin-binding domain. Investigative Ophthalmology and Visual Science $\mathbf{4 5}$ 955-963. 
Ronziere MC, Farjanel J, Freyria AM, Hartmann DJ \& Herbage D 1997 Analysis of types I, II, III, IX and XI collagens synthesized by fetal bovine chondrocytes in high-density culture. Osteoarthritis Cartilage 5 205-214.

Schena M, Shalon D, Heller R, Chai A, Brown PO \& Davis RW 1996 Parallel human genome analysis: microarray-based expression monitoring of 1000 genes. PNAS 93 10614-10619.

Skinner MK, Tung PS \& Fritz IB 1985 Cooperativity between Sertoli cells and testicular peritubular cells in the production and deposition of extracellular matrix components. Journal of Cell Biology $1001941-1947$.
Wu SM, Baxendale V, Chen Y, Pang AL, Stitely T, Munson PJ, Leung MY, Ravindranath N, Dym M, Rennert OM \& Chan WY 2004 Analysis of mouse germ-cell transcriptome at different stages of spermatogenesis by SAGE: biological significance. Genomics $\mathbf{8 4}$ $971-981$.

Received 17 February 2005

First decision 12 April 2005

Revised manuscript received 16 May 2005

Accepted 6 June 2005 